\title{
ASSESSMENT OF FRICTION LOSS TO HORIZONTALLY BUILT FLUID PASSAGES USING ADDITIVE MANUFACTURING
}

\author{
Yi Zhu*, Lei Zhou, Lei Zhang, Cong Zhao, Zimu Wang, Huayong Yang \\ State key laboratory of fluid power and mechatronic systems, Zhejiang University, 38 Zheda Road, 310027 \\ Hangzhou, China \\ * Corresponding author: Tel.: +86 18694580181; E-mail address: yiz@zju.edu.cn
}

\begin{abstract}
Selective laser melting (SLM), is a type of additive manufacturing, which selectively melts a pre-spread layer of metal powders and produce a part by a layer-on-layer manner. SLM has demonstrated a great potential to reduce size and weight in hydraulic manifolds. However, a theoretical base is lacking since friction loss is unclear in a SLMed fluid passage. In this study, various fluid passages without supports, from diameters from $4 \mathrm{~mm}$ to $16 \mathrm{~mm}$, were produced horizontally using SLM. The profile was measured using a 3D scanner and surface roughness was measured using a confocal laser scanning microscope. Friction factor was studied using simulation, experiments, and classical theory. The hydraulic diameter of the SLMed passages is smaller than the design diameter. Surface roughness is extremely high on the top part of the inner wall while the rest part is around $10 \mu \mathrm{m}$. Such trends are irrelevant of passage diameters. Friction factors in SLMed passage is much larger than those predicted using Moody theory, particularly in laminar flow. The transition from laminar flow to turbulent flow appears at a smaller Reynolds number with increased passage diameter. The influence of the profile overweighs that of the surface roughness on friction factor.
\end{abstract}

Keywords: hydraulic manifold; fluid passage; selective laser melting; friction factor

\section{INTRODUCTION}

Selective laser melting (SLM), as one type of metal additive manufacturing (AM), has drawn interests from hydraulic industries as it could make hydraulic components small and light, which is important to the power-to-mass ratio of hydraulic systems [1-3]. One advantage of AM technology lies in the great design freedom of flow paths compared to conventional machining. However, internal supports in the overhang regions of fluid passages are necessary to avoid fabricating defects and failures in SLM according to the residue stress-induced deformation $[4,5]$. In SLM design, fluid passages are often curved in order to reduce size and weight. Therefore, those support structures are impossible to be removed by post-processing due to lack of accessibility [6]. SLM passages should be processed without any internal support structures. On the other hand, fluid passages in a hydraulic manifold are often multi-directional which cannot be all built vertically (i.e. when the passage axis is parallel to the building direction) to avoid internal support structures.

Horizontal fluid passages have the largest overhang regions thus are the most difficult to process. Both large shape deviations and high surface roughness are observed [6,7]. Schmelzle et al. [8] proposed non-circular passages (e.g. diamond, teardrop shapes) in a SLM hydraulic manifold which great reduce the overhang regions. However, ideal circular passages have the highest flow capacity and the least stress concentration on the wall compared to any noncircular passages. The influence of a horizontal SLM fluid passage (without supports) on the friction loss becomes critical in designing SLM hydraulic manifolds.

Some work was performed focusing on SLM cooling channels which do not only consider heat transfer effects but also friction loss [7,9-11]. Snyder et al. [7,9] studied the influence of the building directions on the geometric tolerance and surface roughness, and experimentally measured the resultant friction factor of air flow. The authors found that the building direction greatly influence the dimensional tolerance and 
surface roughness which further affects transition between flow regimes. The horizontal cylindrical channels have the highest friction factor while the vertical channels have the lowest. Stimpson et al. $[10,11]$ studied the characterization of SLM rectangular channels built diagonally using Inconel 718 and CoCr. They analyzed that influence of materials, upskin and downskin surface on the dimensional tolerance and surface roughness. Friction factor was also measured. Results indicated that the friction factor of the SLM channels is higher than that of laminar flow theory in the low Reynolds number while in the turbulent region the difference in the SLM channels is large. A corrected relation between the friction factor and $R_{a} / D_{h}$ was also developed. However, all the work was based on a few types of mini channels (diameter or equivalent diameter $<2 \mathrm{~mm}$ ). Fluid passages used in most hydraulic industries have much larger diameters which are more difficult to build horizontally using SLM. Kamat et al. [6] studied the horizontal built circular, diamond, and teardrop channels with $12 \mathrm{~mm}$ diameter. The shape deviation, particularly on the top of the channels, was found to be critical due to residual stressinduced curling and dross formation in the large overhang regimes. The authors developed an analytical tool to compensate the deformation. However, the high surface roughness and resultant fluid flow were not discussed.

This study presents the friction factor characterization of horizontal SLM hydraulic passages with various diameters. Profile deviation and surface roughness were measured. Friction factor was investigated using computational fluid dynamics simulation and experiments. The work helps to develop design criteria for novel hydraulic components using SLM.

\section{METHODS}

\subsection{Horizontal fluid passage fabrication}

The fluid passage samples with various diameters $(4,6,8,10,12,14,16 \mathrm{~mm})$ were fabricated using SLM. Those diameters were selected since they are commonly used in industrial hydraulic systems. The length of each passage is $130 \mathrm{~mm}$. The wall thickness of each passage is $2 \mathrm{~mm}$. The building orientation is shown in Fig. 1. All fluid passages were built using the same height and design of external support structures on the bottom. No internal support structures were added to any fluid passages. Some samples were also fabricated using the same design and procedure with a short length of $10 \mathrm{~mm}$ for surface and profile measurements. The SLM fabrication was performed using a Renishaw (UK) AM250 laser melting unit comprised of an SPI redPOWER $200 \mathrm{~W}$ ytterbium fiber laser, an automatic powder layering system, an argon gas protection system, and a process control system. The laser operated with a $70 \mathrm{~mm}$ focused beam diameter at a $1071 \mathrm{~nm}$ wavelength. A schematic of the SLM system is shown in Fig. 2. It is noted that the roller recoats the powder layer from back to forth (where the observation window is) in the Renishaw system which differs from other systems. 316L stainless steel was used in the work. The powder characterization was introduced in our previous work [12-14]. The process parameters used in the study was listed in Table 1.

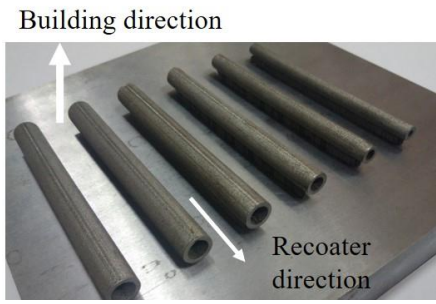

Figure 1: Building orientation of the fluid passages

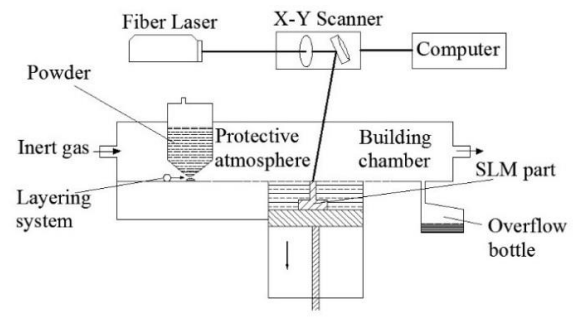

Figure 2: A schematic of SLM system used in the work [13].

Table 1: Process parameters used in the work

\begin{tabular}{llllll}
\hline $\begin{array}{l}\text { Laser } \\
\text { power }\end{array}$ & $\begin{array}{l}\text { Layer } \\
\text { thickness }\end{array}$ & $\begin{array}{l}\text { Point } \\
\text { distance }\end{array}$ & $\begin{array}{l}\text { Exposure } \\
\text { time }\end{array}$ & $\begin{array}{l}\text { Hatch } \\
\text { space }\end{array}$ & $\begin{array}{l}\text { Scan } \\
\text { strategy }\end{array}$ \\
\hline 200 & $50 \mu \mathrm{m}$ & $65 \mu \mathrm{m}$ & $80 \mu \mathrm{s}$ & 80 & Stripes \\
$\mathrm{W}$ & & & & $\mu \mathrm{m}$ & \\
\hline
\end{tabular}




\subsection{Measurements of profile and surface topography of fluid passages}

Though all the fluid passage samples were designed with a circular-shape cross section, the as-built samples have geometry errors caused by curling and dross formation on the overhang regimes. Moreover, the inner wall surface of the passage is very rough, particularly on the top part. The profile of each fluid passage sample was measured using an optical 3D scanner (OKIO3M, Shining 3D Ltd, China) as shown in Fig. 3. The 3D scanner has measuring accuracy of 0.005 $\mathrm{mm}$ and a scanning distance of $0.04 \mathrm{~mm}$. A commercial software Geomagic Control X was used to analyze the measured profiles of all fluid passages. Hydraulic diameter, which is commonly used to evaluate the flow capacity of non-circular passages, was calculated using profile data. Thus, an optical 3D scanner is used in the work which is not only efficient in measuring profiles of many samples but also low cost compared to the commonly used industrial CT scanner for evaluating the quality of SLM parts. The surface roughness measurements were performed using a confocal laser scanning microscopy (VK150, Keyence, Japan) on four spots of each sample: top, bottom, left, and right, which are shown in Fig. 4. It is noted that those extra samples were produced under the same conditions as the fluid passages. Each measurement was performed by scanning a $1 \mathrm{~mm}$ $\times 1.39 \mathrm{~mm}$ area. Such measurement was repeated three times on each spot of the sample along the axial direction. $S_{a}$, a $3 \mathrm{D}$ surface roughness parameter represents arithmetical mean height, was calculated while the $\mathrm{S}$ filter was set to $5 \mu \mathrm{m}$ and the L filter was set to $2 \mathrm{~mm}$. It is noted that $S_{a}$ is the extension of $R_{a}$ (arithmetical mean height of a line, 2D) to a 3D surface.

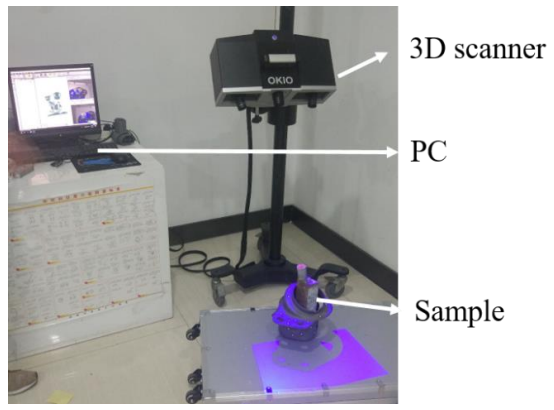

Figure 3: Scanning the sample profile using a 3D scanner (photo taken by L. Zhang).

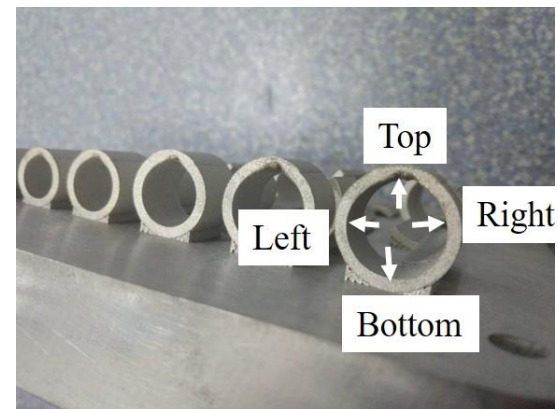

Figure 4: The location of surface roughness measurements by white arrows.

\subsection{Simulation}

The subject of simulation is to formulate a full map between friction factor, passage diameter, and Reynold number. However, the accuracy of the simulation in terms of friction factor greatly depends on the mesh generation which may cause extremely long computational time. Moreover, the input to the simulation, e.g., the profile data and surface roughness, has simplified compared to the real situation. Therefore, the simulation in the study is mainly used to give a reference and calculate the local loss in the rig in addition to the friction loss in the passage.

The simulation was performed using CFX in ANSYS. Taken the $10 \mathrm{~mm}$ (design diameter) SLMed fluid passage for example, unstructured mesh was designed to obtain a ratio between the element size and the minimum passage diameter to be 0.04 . The boundary layer was meshed near the internal walls where the friction loss is mainly generated. The parameters in inflation were set to $0.01 \mathrm{~mm}$ as the first layer thickness, 26 as the maximum layers, and 1.05 as the growth rate. Table 2 shows the number of nodes and elements used in the simulation. Simulation of fluid passages with other diameters was run on similar conditions.

Table 2: Mesh characteristics for the CFD analysis with a "mesh ratio" of 0.04

\begin{tabular}{ll}
\hline $\begin{array}{l}\text { Mesh Ratio: Element Size/Minimum } \\
\text { Channel Diameter }\end{array}$ & 0.04 \\
\hline Number of nodes & $3,476,468$ \\
Number of elements & $15,149,160$ \\
\hline
\end{tabular}

The fluid used in the simulation is $46 \#$ hydraulic oil, which has a kinematic viscosity at $40^{\circ}$ of 46 cSt and a density of $860 \mathrm{~kg} / \mathrm{m} 3$. The imposed boundary conditions were the flow rate used in 
the experiments at the inlet and $1 \mathrm{MPa}$ pressure at the outlet. The measured passage profile and surface roughness were given as inputs to the model in the simulation. In addition, $\mathrm{k}-\omega$ model was used as the turbulence model because it is a model for small Reynolds number, which performs well for hydraulic systems [15].

In the numerical analyze, a fully developed flow was assumed in the measuring length. A finer mesh was also used to generate meshes and run the simulation. Results indicated that the pressure difference between using current mesh (with mesh ratio 0.04) and a finer mesh (with mesh ratio $<0.04$ ) is below the accuracy of the sensors used for measuring pressure loss in the experiments $(0.012 \mathrm{MPa})$. This has confirmed that the current mesh generated does not decrease the simulation accuracy with a reasonable computational time.

\subsection{Experimental details}

The pressure loss of the fluid passages with various diameters was measured using a customized test rig as shown in Fig. 5. It can be found that the measured pressure loss $P_{s}$ includes the actually pressure loss along the SLM passage $P_{0}$ and the local loss in the measuring equipment. The local loss is calculated in the abovementioned simulation.
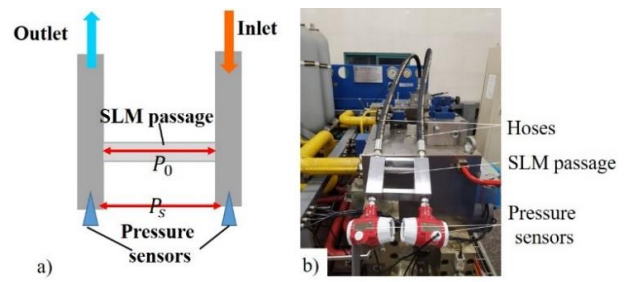

Figure 5: a) Schematic of the test rig; b) a photo showing the test rig.

The friction factor 1 can be calculated using Equation 1:

$$
\lambda=\frac{2 d P_{0}}{l \rho v^{2}}
$$

where $\mathrm{d}$ is the hydraulic diameter; 1 is the passage length; $\rho$ is the density of the fluid; $v$ is the average velocity of the fluid.

\section{RESULTS}

\subsection{Measuring results}

The hydraulic diameter results are shown in
Fig. 6. The relative discrepancy between the design diameter and hydraulic diameter is also illustrated. The results clearly indicate that the geometry errors decrease with increased passage diameters. The diameter difference between the design and the fabricated passages does not vary much.

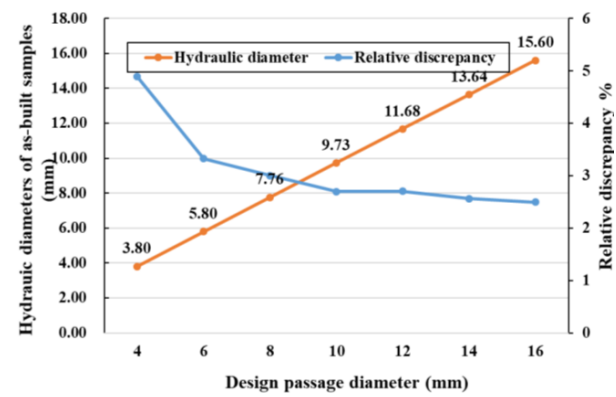

Figure 6: Results of hydraulic diameters

Surface roughness of the four location, top, right, left, and bottom, in each fluid passage are shown in Fig. 7. The roughness on the top part is extremely high, from approximately 60 to $80 \mu \mathrm{m}$. The surface roughness of the rest, right, left, and bottom, are approximately $10 \mu \mathrm{m}$ with slight variations, which does not show dependency on the passage diameters. From empirical friction loss calculation of laminar flow, the surface roughness is considered to be below $20 \mu \mathrm{m}$ using conventional machining for any metal fluid passages in hydraulic systems. Therefore, the surface roughness of the top part creates large difference compared to the conventionally machined fluid passages.

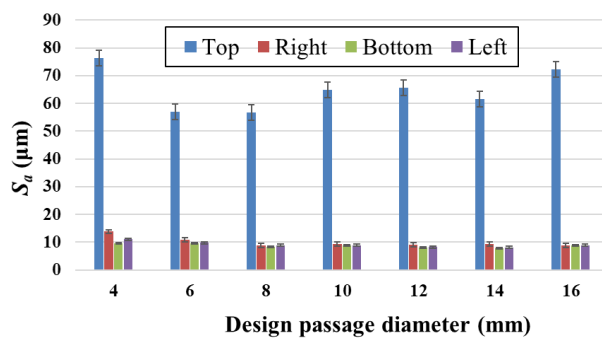

Figure 7: Results of surface roughness

\subsection{Friction factor results}

Due to the manufacturing discrepancy, the dimensional accuracy of the passage profile varies which is also observed in [7]. Therefore, the passage diameter (or hydraulic diameter) $d$ used in Reynolds number is not a constant for 
SLMed fluid passages. The variation is related to some manufacturing factors, e.g., building directions, passage structures, and process parameters. Since the influence of the passage diameter cannot be fully represented in the Reynolds number, the friction factor curve of each fluid passage is plotted. A 3D map of the friction factor versus passage diameter and Reynold number is shown in Fig. 8. The trends, in general, is similar to the classical Moody diagram. However, it can be seen that the transition from laminar flow to turbulent flow appears at a smaller Reynold number with increased passage diameter. The transition is highlighted using a red dot in Fig. 8. The friction factor seems to increase with increased passage diameter which conflicts the classical theory. It may due to the fact that with increased diameter, the friction loss greatly reduces, while the local loss becomes a dominating factor in the total pressure loss measurements. A future work needs to be performed to modify the test rig.

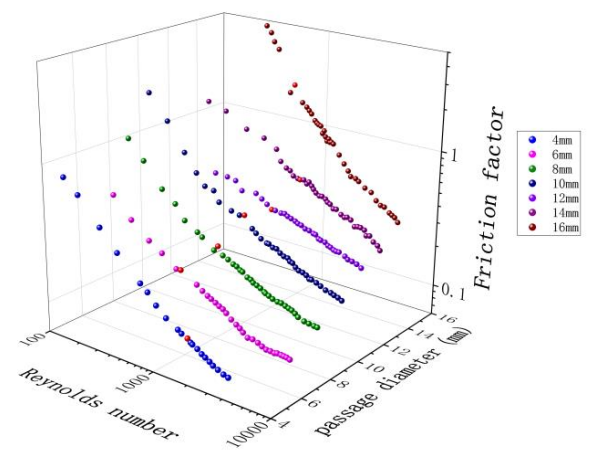

Figure 8: 3D map of friction factor.

Figs. 9-11 show the friction factors obtained from experimental results (blue dots), simulation results (red dots), and classical theory (black solid line for laminar flow and black dashed line for turbulent flow) in three SLMed fluid passages with diameters of 6,8 , and $10 \mathrm{~mm}$. In the classical theory, $75 / \operatorname{Re}$ (empirical equation) is used to calculate the friction factor in laminar flow and Blasius equation is used to calculate the friction factor in turbulent flow inside a smooth passage. Compared the experimental results with classical theory of smooth passages, the friction factor of the SLMed passages is much higher. The transition from laminar to turbulent flow of SLMed passages appears at $\operatorname{Re} 900$ which is much smaller than the classical theory. The friction factors obtained from simulations are between the experimental results and the classical theory. With increased passage diameter, the difference between simulation and experimental results decreases.

The experimental results are also plotted with corrected empirical equation (green solid line) and Colebrook equation (green dashed line). The Colebrook equation can be written as:

$$
\frac{1}{\sqrt{\lambda}}=-2 \lg \left(\frac{\Delta}{3.7 d}+\frac{2.51}{\operatorname{Re} \sqrt{\lambda}}\right)
$$

where $\Delta$ is the roughness parameter. In the laminar flow, the corrected empirical equation indicates a large slope, which becomes even steeper with increased fluid diameters. It is noted that a constant hydraulic diameter in Reynolds number does not fully reflect the actual diameter in a SLMed passage due to manufacturing discrepancy. Thus, fluid passages with different diameters have different equations in the laminar flow. In the turbulent flow, Sa value of the top surface has been selected as the roughness parameter $\Delta$. However, quite large discrepancy can be found between experimental results and Colebrook equations. It is noted the difference between the two becomes even greater with a reduced roughness parameter.

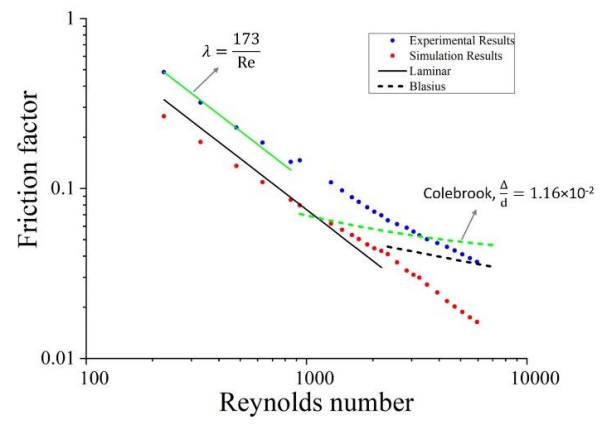

Figure 9: Friction factor comparison of a $6 \mathrm{~mm}$ SLMed fluid passage. 


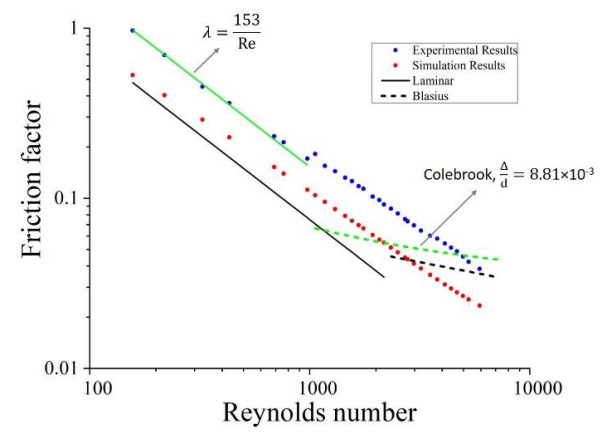

Figure 10: Friction factor comparison of a $8 \mathrm{~mm}$ SLMed fluid passage.

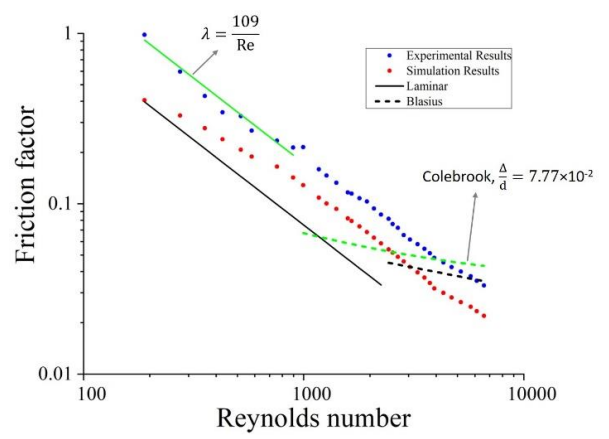

Figure 11: Friction factor comparison of a $10 \mathrm{~mm}$ SLMed fluid passage.

\section{DISCUSSION}

As already pointed out by many researchers [1618], a large overhang region is subjected to dross formation and curling on the downskin part using SLM technology. As a guideline which is widely used in many SLM systems, manufacturing failure may appear for overhang regions larger than $5 \mathrm{~mm}$. Horizontal fluid passage without supports is one type of overhang structures. According to the produced sample, fluid passage with $16 \mathrm{~mm}$ diameter can be fabricated without supports. A $20 \mathrm{~mm}$ diameter passage can also be fabricated which is not mentioned in this study. Therefore, the manufacturability of horizontal fluid passages without supports is much beyond the expectation of common design guidelines. On the other hand, dimensional error in the profile seems to be irrelevant to the passage diameter. Moreover, surface roughness shows very similar characteristics among all SLMed fluid passages. Surface roughness on the downskin part is extremely high compared to the other parts, and the roughness value is irrelevant to the passage diameter. Thus, the influence of profile and surface roughness on the fluid flow is more critical for small-diameter passages.

Figs. 9-11 indicate that the SLMed fluid passages do not behave like normal rough channels. Classical theory does not give acceptable results compared to the experiments. The influence of the profile on the friction factor can be clearly seen in the laminar flow. The hydraulic diameter in Reynolds number cannot fully reflect the manufacturing discrepancy. Therefore, each fluid passage has different empirical equation in laminar flow. A more accurate method is required to assess the influence of the profile. Both profile and surface roughness affect the friction factor in turbulent flow. However, large discrepancy still exists even a downskin roughness is used in the Colebrook equation. Therefore, the influence of the profile on the friction factor in turbulent flow is great which also needs a further study.

\section{CONCLUSIONS}

Various fluid passages from $4 \mathrm{~mm}$ to $16 \mathrm{~mm}$ without supports were produced horizontally using SLM. Friction factor in the fluid passages is studied using simulation, experiments, and classical theory. The effect of profile and surface roughness is discussed. The study allows the following conclusions to be drawn:

- Curling was observed for on the top part of all SLMed passages. The hydraulic diameter is smaller than the design diameter.

- Surface roughness is extremely high on the top part, which is at least four times higher than the rest part of the inner wall.

- Friction factors in SLMed passage is much larger than those predicted using Moody theory, particularly in laminar flow.

- The transition from laminar flow to turbulent flow appears at a smaller Reynolds number compared to the classical theory. For SLMed fluid passages, the transition appears at a smaller Reynolds number with increased passage diameters.

- The influence of the profile overweighs that of the surface roughness on friction factor.

Many future works are required: 1 to improve manufacturing quality by optimizing process 
parameters and to study the friction factor of improved ones; 2 to upgrade the test rig; 3 to study the manufacturing and friction factor of curved fluid passages in different building directions. A design tool of SLMed fluid passage is the final subject which cannot achieved without a friction factor model.

\section{REFERENCES}

[1] Https://www.moog.com/3dmetal/index.html, Moog additive overview, (n.d.).

[2] Https://www.renishaw.com/en/hydraulic-blockmanifold-redesign-for-additive-manufacturing-38949, Hydraulic block manifold redesign for additive manufacturing, (n.d.).

[3] Https://www.aidro.it/3d-metal-printing.html, Aidro hydraulics and 3D printing, (n.d.).

[4] B. Vandenbroucke, J. Kruth, Selective laser melting of biocompatible metals for rapid manufacturing of medical parts, Rapid Prototyping Journal. 13 (2007) 196-203. doi: $10.1108 / 13552540710776142$.

[5] P. Mercelis, J.P. Kruth, Residual stresses in selective laser sintering and selective laser melting, Rapid Prototyping Journal. 12 (2006) 254-265. doi:10.1108/13552540610707013.

[6] A.M. Kamat, Y. Pei, An analytical method to predict and compensate for residual stressinduced deformation in overhanging regions of internal channels fabricated using powder bed fusion, Additive Manufacturing. 29 (2019) 100796. doi:10.1016/j.addma.2019.100796.

[7] J.C. Snyder, C.K. Stimpson, K.A. Thole, D.J. Mongillo, Build Direction Effects on Microchannel Tolerance and Surface Roughness, Journal of Mechanical Design. 137 (2015) 111411. doi:10.1115/1.4031071.

[8] J. Schmelzle, E. V Kline, C.J. Dickman, E.W. Reutzel, G. Jones, T.W. Simpson, (Re)Designing for Part Consolidation: Understanding the Challenges of Metal Additive Manufacturing, Journal of Mechanical Design. 137 (2015) 111404. doi:10.1115/1.4031156.

[9] J.C. Snyder, C.K. Stimpson, K.A. Thole, D. Mongillo, Build Direction Effects on Additively Manufactured Channels, Journal of Turbomachinery. $138 \quad$ (2016) 051006. doi:10.1115/1.4032168.

[10] C.K. Stimpson, J.C. Snyder, K.A. Thole, D. Mongillo, Scaling Roughness Effects on Pressure Loss and Heat Transfer of Additively Manufactured Channels, Journal of
Turbomachinery. $139 \quad$ (2016) 021003. doi:10.1115/1.4034555.

[11] C.K. Stimpson, J.C. Snyder, K.A. Thole, D. Mongillo, Roughness Effects on Flow and Heat Transfer for Additively Manufactured Channels, Journal of Turbomachinery. 138 (2016) 051008. doi:10.1115/1.4032167.

[12] Y. Zhu, T. Peng, G. Jia, H. Zhang, S. Xu, H. Yang, Electrical energy consumption and mechanical properties of selective-lasermelting-produced 316L stainless steel samples using various processing parameters, Journal of Cleaner Production. 208 (2019) 77-85. doi:10.1016/j.jclepro.2018.10.109.

[13] Y. Yang, Y. Zhu, M.M. Khonsari, H. Yang, Wear anisotropy of selective laser melted 316L stainless steel, Wear. 428-429 (2019) 376-386. doi:10.1016/j.wear.2019.04.001.

[14] Y. Zhu, Y. Yang, X. Mu, W. Wang, Z. Yao, H. Yang, Study on wear and RCF performance of repaired damage railway wheels: Assessing laser cladding to repair local defects on wheels, Wear. 430-431 (2019) 126-136. doi:10.1016/j.wear.2019.04.028.

[15] B. Zardin, G. Cillo, M. Borghi, A. D'Adamo, S. Fontanesi, Pressure losses in multiple-elbow paths and in V-bends of hydraulic manifolds, Energies. 10 (2017). doi:10.3390/en10060788.

[16] J. Kranz, D. Herzog, C. Emmelmann, Design guidelines for laser additive manufacturing of lightweight structures in TiAl6V4, Journal of Laser Applications. 27 (2015) S14001. doi:10.2351/1.4885235.

[17] D. Wang, Y. Yang, Z. Yi, X. Su, Research on the fabricating quality optimization of the overhanging surface in SLM process, International Journal of Advanced Manufacturing Technology. 65 (2013) 14711484. doi:10.1007/s00170-012-4271-4.

[18] D. Thomas, The Development of Design Rules for Selective Laser Melting, University of Wales Institute, Cardiff, 2009. 